\title{
SELF-RELEVANCE PREDICTS THE AESTHETIC APPEAL OF REAL AND SYNTHETIC ARTWORKS GENERATED VIA NEURAL STYLE TRANSFER
}

\author{
A PREPRINT
}

\author{
Edward A. Vessel \\ Department of Neuroscience \\ Max Planck Institute for Empirical Aesthetics \\ Frankfurt (Main), Germany \\ ed.vessel@ae.mpg.de
}

\author{
Laura Pasqualette \\ Psychology Department \\ Friedrich-Alexander University Erlangen-Nürnberg \\ Erlangen, Germany
}

\author{
Cem Uran \\ Ernst Strungmann Institute \\ Frankfurt (Main), Germany
}

\author{
Sarah Koldehoff \\ Department of Neuroscience \\ Max Planck Institute for Empirical Aesthetics \\ Frankfurt (Main), Germany
}

\author{
Martin Vinck \\ Ernst Strungmann Institute \\ Frankfurt (Main), Germany
}

Compiled: October 1, 2021

\begin{abstract}
What determines the aesthetic appeal of artworks? Recent work suggests that aesthetic appeal can to some extent be predicted from a visual artwork's image features. Yet, a large fraction of variance in aesthetic ratings remains unexplained and may relate to individual preferences. We hypothesized that an artwork's aesthetic appeal depends strongly on self-relevance. In a first experiment, observers viewed real artworks and rated them for aesthetic appeal and self-relevance. Aesthetic appeal was positively predicted by self-relevance. In a second experiment, we developed a method to create synthetic, self-relevant artworks, by using deep neural networks that transferred the style of existing artworks to photographs. Style transfer was applied to self-relevant photographs which were identified based on autobiographical memories, self-identity, interests, common activities and preferences. Self-relevant, synthetic artworks were rated as more aesthetically appealing than matched control images, at a level similar to real artworks. Thus, self-relevance is a key determinant of aesthetic appeal, independent of artistic skill and image features.
\end{abstract}

\section{Introduction}

Experiences with artwork can impact us deeply. They can move us emotionally and inspire us creatively [1]. They can also be transformative, opening up new ways of looking at ourselves and the world around us. What determines the aesthetic appeal of an artwork, and differences in aesthetic experiences between individuals?

Recently it has been shown that a linear combination of low- and high-level image features predicts, on average, around $20 \%$ of variance in observer ratings [2]. A comparable performance (16.4\%) was obtained by a deep neural network trained to predict aesthetic ratings [2]. These findings suggest that a large fraction of variance in aesthetic ratings across individuals remains unexplained. Indeed, previous studies have estimated that only about $10 \%$ of the reliable variance in aesthetic ratings of artworks can be accounted for by a "shared taste" factor [3, 4], i.e. the remaining 90\% differs from person to person.

We hypothesized that a key factor explaining aesthetic appeal is the capacity of an artwork to deeply impact a person depending on whether it speaks to one's individually lived experience. Does the artwork resonate with one's sense 
of self and worldview? This can be captured by the concept of self-relevance, which reflects the extent to which something relates to a person's self-schema: their self-perception, their past experiences and their personal and social identity [5, 6]. One's self-schema is chronically accessible [7], is known to be accessed when evaluating others [8,-10], and effects memory encoding [11,-13]. Several existing frameworks for understanding aesthetic experiences suggest that self-relevance is important for aesthetic appeal [14, 15]. Furthermore, brain imaging work suggests that the default-mode network, which supports central aspects of self-referential mentation [16-18], may play a role in moving aesthetic experiences with artwork [19-22]. Here, we performed a set of experiments to directly investigate the influence of self-relevance on aesthetic appeal.

In two experiments, we tested whether self-relevance predicts aesthetic appeal. In a first experiment, we correlated aesthetic ratings with self-relevance assessments, and established that there is a strong relationship between selfrelevance and aesthetic appeal. We then developed a new method to directly manipulate self-relevance by using a deep learning technique known as 'style transfer' [23]. By transferring the styles of existing artworks to pictures using neural networks, we created novel artworks that had high self-relevance to particular individuals. These were then rated by participants for aesthetic appeal, intermixed with artworks created for a different observer and a set of real artworks. We further analyzed which aspects of self-relevance accounted for the observed effects on aesthetic appeal.

\section{Results}

\section{Ratings of self-relevance strongly predict aesthetic appeal}

In a first experiment, 33 participants viewed 148 artworks and rated them for aesthetic appeal (e.g., "How much does this artwork move you?") and self-relevance (e.g. "How self-relevant is this artwork for you?") in separate blocks. Twenty of the artworks were viewed and rated again on both measures to allow for a computation of participant-specific test-retest reliability scores.

Individual ratings of aesthetic appeal were modeled as a function of individual ratings of self-relevance using a linear mixed model (LMM), with participant-specific intercepts and slopes and image-specific intercepts (see Methods). Observers differed in which paintings they found to be aesthetically appealing or self-relevant ("mean-minus-one" agreement metric $M M 1=0.40$ and 0.39 respectively; $24 \%$ and $18 \%$ of repeatable variance explained by "shared taste" ). Yet despite this variability, individual ratings of whether a painting was judged to be self-relevant were strongly predictive of aesthetic ratings (Fig. 3a; slope $=0.36,95 \%$ CI $[0.30,0.42], \mathrm{t}[32.3]=12.4, p=8.3 \times 10^{-14}$ ). This relationship was even stronger in the "retest" block (slope $=0.56,95 \% \mathrm{CI}[0.48,0.63], \mathrm{t}[130]=15.9, p=3.4 \mathrm{x}$ $10^{-32}$ ) suggesting that calling explicit attention to the dimension of self-relevance further increased the already strong relationship between these two constructs (though we note that retest blocks included only a subset of 20 images).

\section{Self-relevant artworks generated using neural 'style-transfer' are more aesthetically appealing than matched other-relevant artworks}

The results of the first experiment suggest that self-relevance is an important determinant of aesthetic appeal. However, because of the correlational nature of that analysis, it is difficult to isolate the influence of self-relevance. Thus, in a second experiment, we sought to specifically manipulate the self-relevance of artworks in a controlled manner to test its influence on rated aesthetic appeal. To that end, we created self-relevant artworks with a deep convolutional neural network (dCNN) to implement style transfer (Fig. 1a). By using style transfer, the artistic style of an existing artwork was combined with the content of a photograph to create a new artwork with new content, but the same style. For each participant, we thereby created custom artworks that were well controlled for both content and style, without having to explicitly ask participants about self-relevance. Novel artworks with personally relevant content were created by applying style transfer to images from online sources that reflected each participant's unique responses to a questionnaire. This questionnaire asked about specific autobiographical memories, self-identity, interests, common activities and preferences ("Cultural Background and Lifestyle Questionnaire", see Methods). For example, one participant wrote about a particularly memorable vacation to Helsinki; an image of a prominent central landmark in Helsinki was combined with a colorful artistic style to generate a novel artwork that reflected a memorable moment specific to that participant. In contrast, for a matched participant who expressed no particular link to Helsinki, the same artwork held no special relevance.

A total of 45 observers participated in the two-session online study. In the first session the participants were asked a series of questions to identify self-relevant photographs. In the second session they rated a set of artworks on several measures. In four blocks, the observers viewed 80 artworks and rated each of them on a single question. Both in Block 1 (test) and Block 2 (retest) observers gave ratings for aesthetic appeal. In Block 3 observers rated the artworks for self-relevance. In Block 4, participant indicated whether the artwork was either "definitely recognized", familiar or unfamiliar (see Methods). The artworks belonged to one of four conditions (Fig. 1b): (1) self-relevant (SR) artworks 


\section{A Participant Responses

\begin{tabular}{|c|}
\hline Cultural and Lifestyle Questionnaire \\
\hline $\begin{array}{l}\text { 1. What was your most remarkable } \\
\text { holiday until now? }\end{array}$ \\
\hline $\begin{array}{l}\text { The most impressive vacation so far was } \\
\text { when I traveled alone from Helsinki }-> \\
\text { Turku } \rightarrow>\text { Oulu } \rightarrow>\text { Haparanda }->\text { Umea }-> \\
\text { Stockholm }->\text { Copenhagen. The clear air } \\
\text { with the incredible landscape and the } \\
\text { various architectural features was } \\
\text { impressive. }\end{array}$ \\
\hline $\begin{array}{l}\text { 2. If you could recommend a place to } \\
\text { visit in your homeland, what would it be? }\end{array}$ \\
\hline $\begin{array}{l}\text { 3. What social, cultural, or religious } \\
\text { groups do you identify with? }\end{array}$ \\
\hline
\end{tabular}

B

Conditions: (20 images each)

\section{Content Source}
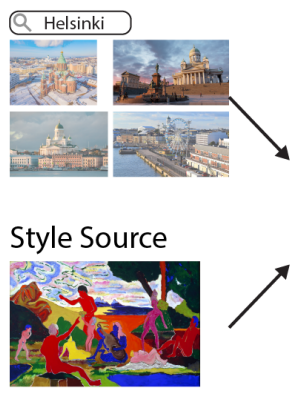

Style Transfer Network

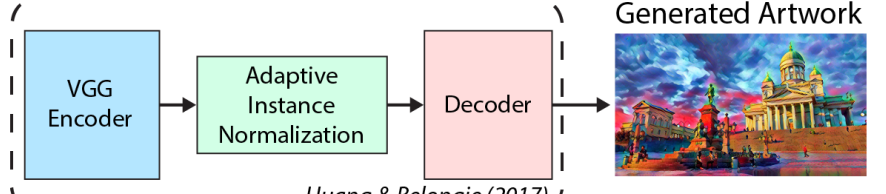

( _ _ _ _ _ Huang요 Belongie (2017)

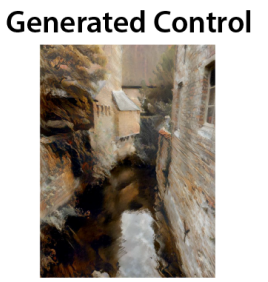

Real Artworks

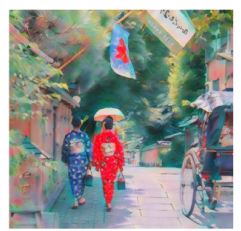

Customized for participants, in pairs

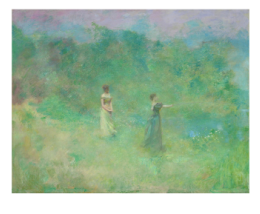

Figure 1: Generating self-relevant artworks using a "style transfer" convolutional neural network. a) In a first session, participants responded to a 'Cultural Background and Lifestyle Questionnaire' that inquired about specific autobiographical memories, aspects of identity, interests, preferences and common activities. Images were then sourced from the internet that contained content relevant to each observer's individual responses. A style of an existing artwork was then transferred to the image using a style transfer network based on [24] that consisted of an encoder network, adaptive instance normalization and a decoder network, resulting in a new synthetic artwork with customized content. b) In a second session, observers were shown artworks from 4 conditions: (1) self-relevant (SR) artworks custom generated based on their questionnaire responses, (2) other-relevant (OR) artworks generated for a matched participant, (3) a control set of generated artworks, and (4) a set of real artworks. There were 20 artworks in each condition.

generated based on their responses to the questionnaire; (2) other-relevant (OR) artworks that were generated for a matched participant; (3) a set of generated control (GC) artworks shown to all participants; or (4) a selection of real artworks (RA), also shown to all participants.

We first validated our methodology by analyzing whether participants indeed rated the SR artworks as more selfrelevant. For this we used a LMM with self-relevance modeled as a function of stimulus condition with participants as a random factor (Fig. 2a). Artworks from the self-relevant category were indeed rated as significantly more selfrelevant than all other categories (SR vs. OR estimate $=0.19,95 \% \mathrm{CI}[0.16,0.21], \mathrm{t}[3160]=13.0, p=1.3 \times 10^{-37}$ ) Additionally, real artworks were rated as less self relevant than the control generated artworks (RA vs. CG estimate $=-0.031,95 \%$ CI $[-0.059,-0.003], \mathrm{t}[3160]=-2.2, p=0.031)$, potentially on account of the fact that the real artwork category contained several abstract works, while the generated artworks contained representational content such as buildings, objects and landscapes. Other-relevant artworks were also rated as more self-relevant than the control generated artworks (OR vs CG estimate $=0.064,95 \% \mathrm{CI}[0.036,0.092], \mathrm{t}[3160]=4.5$, post-hoc comparison adjusted $p=1.71 \times 10^{-5}$ using Holm method). Content that was selected as self-relevant for a given participant had a tendency to be more self-relevant for matched participants than the control generated artworks, likely reflecting some degree of general shared experience across participants.

Next, we analyzed how aesthetic ratings differed between self- and other-relevant artworks. Artworks generated from self-relevant content were rated as significantly more appealing than matched other-relevant artworks (SR vs. OR estimate $=0.071,95 \%$ CI $[0.048,0.095], \mathrm{t}[3160]=5.9, p=3.8 \times 10^{-9} ;$ LMM with aesthetic appeal modeled as a function of stimulus condition with participants as a random factor). In addition, although real artworks were rated as significantly more aesthetically appealing than the control generated artworks (RA vs. CG estimate $=0.046,95 \%$ CI $[0.023,0.070], \mathrm{t}[3160]=3.8, p=0.00013)$, the self-relevant generated artworks recovered this difference, even being slightly preferred to the real artworks on average (SR vs. RA estimate $=0.014,95 \%$ CI $[-0.0097,0.038], \mathrm{t}[3160]=1.2$, $p=0.25$; not significant). Thus, generating new artworks with self-relevant content was highly effective at increasing 
A

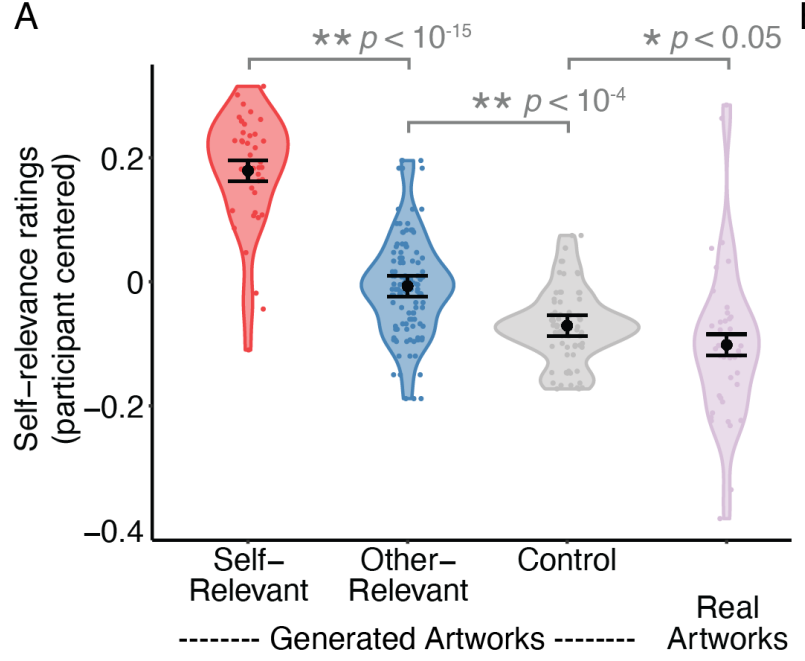

B

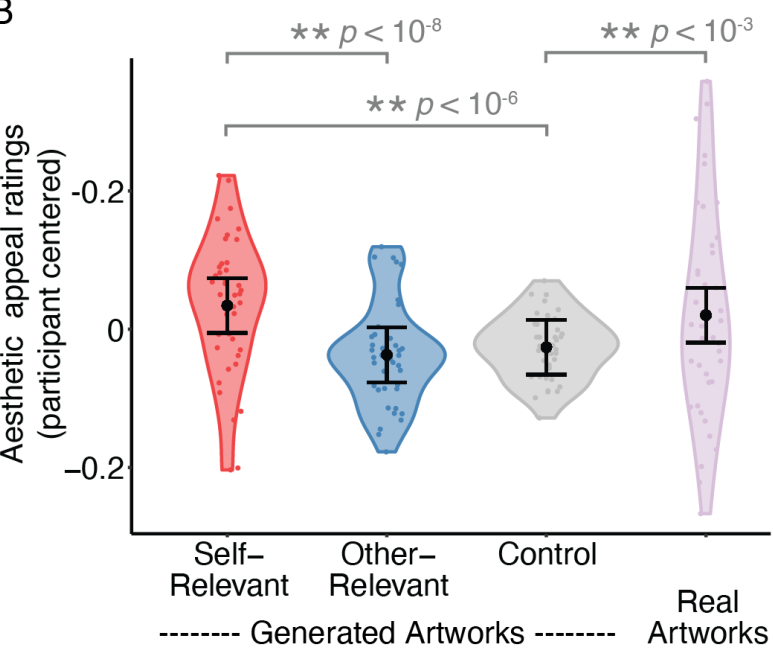

Figure 2: Effect of stimulus condition on ratings of a) self-relevance and b) aesthetic appeal in Exp. 2. Colored dots show average ratings for individual participants in each condition, after centering by participant, and shaded area indicates data density, smoothed with a gaussian kernel and trimmed. Black dot and error bars indicate the mean and $95 \%$ confidence intervals. Gray horizontal brackets indicate significance. $\mathrm{N}=40$ participants.

aesthetic appeal in a manner that was independent of the artistic skill that characterized the real artworks. Hence the presence of content that related to a participant's lived experience, identity and interests predicted stronger aesthetic appeal.

We then examined how aesthetic appeal related to ratings of self-relevance at the level of individual artworks. To this end we predicted participant ratings of aesthetic appeal from their ratings of self-relevance, for all artworks from all four conditions. We found that the effect of self-relevance on aesthetic appeal was very strong (Fig. 3b): A change from not self-relevant to highly self-relevant increased aesthetic ratings by 35 points on a 100 point scale (slope $=$ 0.35 for data rescaled to between 0 and $1,95 \%$ CI [0.32 0.37], t [2940] $=25, p=4.1 \times 10^{-122}$ ). This finding further supports the conclusion that self-relevance is a major determinant of aesthetic appeal.

We wondered if the effect of self-relevance might have been mediated by familiarity. To investigate this, we predicted aesthetic appeal from ratings of both self-relevance and familiarity (with random intercepts for participant, see Methods). We found that paintings that were "definitely recognized" or "familiar" were more preferred than "unfamiliar" paintings (Fig. 3c; slope $=0.086,95 \%$ CI $[0.063,0.11]$, t[2795] $=7.2, p=7.9 \times 10^{-13}$ ), and that "definitely recognized" paintings were slightly more preferred than "familiar" paintings (slope $=0.045,95 \% \mathrm{CI}[0.010,0.080], \mathrm{t}[2855]$ $=2.5, p=0.011$ ). However, self-relevance was still a very strong predictor (slope $=0.30,95 \% \mathrm{CI}[0.22,0.37], \mathrm{t}[18.9]=$ $8.1, p=1.4 \times 10^{-7}$ ). A formal mediation analysis revealed that familiarity was able to explain only a small fraction of the self-relevance effect (Fig. 3d; average causal mediation effect $=0.04,95 \% \mathrm{CI}[0.03,0.05]$; remaining direct effect of self-relevance on aesthetic appeal $=0.33,95 \% \mathrm{CI}[0.30,0.35])$. Thus, we conclude that the effect of self-relevance was not mediated by familiarity.

\section{Artworks reflecting core concepts of one's self-construct were more appealing}

Self-relevance can relate to different aspects of the self-construct, such as memories, interests and personal identity. We therefore sought to determine which aspects of self-relevance influenced aesthetic appeal. To this end, we reclassified the self-relevant artworks into several subclasses based upon which questionnaire item they were derived from. Based on this, we identified five different classes of generated artworks reflecting: (1) specific autobiographical memories; (2) interests; (3) aspects of personal identity; (4) common activities; and (5) explicitly expressed preferences. In addition we identified a sixth "mixed" class. Ratings of aesthetic appeal for the 20 participant-specific generated artworks in the self-relevant (SR) condition were modeled as a function of these six classes (see Methods). Artwork classes that were most associated with higher aesthetic ratings (Fig. 4) were: autobiographical memories (slope $=0.10,95 \%$ CI $[0.07$, $\left.0.13], \mathrm{t}[1555]=6.0, p=3.2 x 10^{-9}\right)$, identity ( preferences (slope $=0.11,95 \% \mathrm{CI}[0.06,0.16], \mathrm{t}[1556]=4.4, p=1.4 \times 10^{-5}$ ) and interests (slope $=0.051,95 \% \mathrm{CI}$ $[0.0002,0.10], \mathrm{t}[1557]=2.0, p=0.050)$. Artworks that related to common activities, however, were not associated with higher aesthetic ratings (slope $=-0.017,95 \% \mathrm{CI}[-0.09,0.06], \mathrm{t}[1558]=-0.46, p=0.64$ ), nor were artworks in 

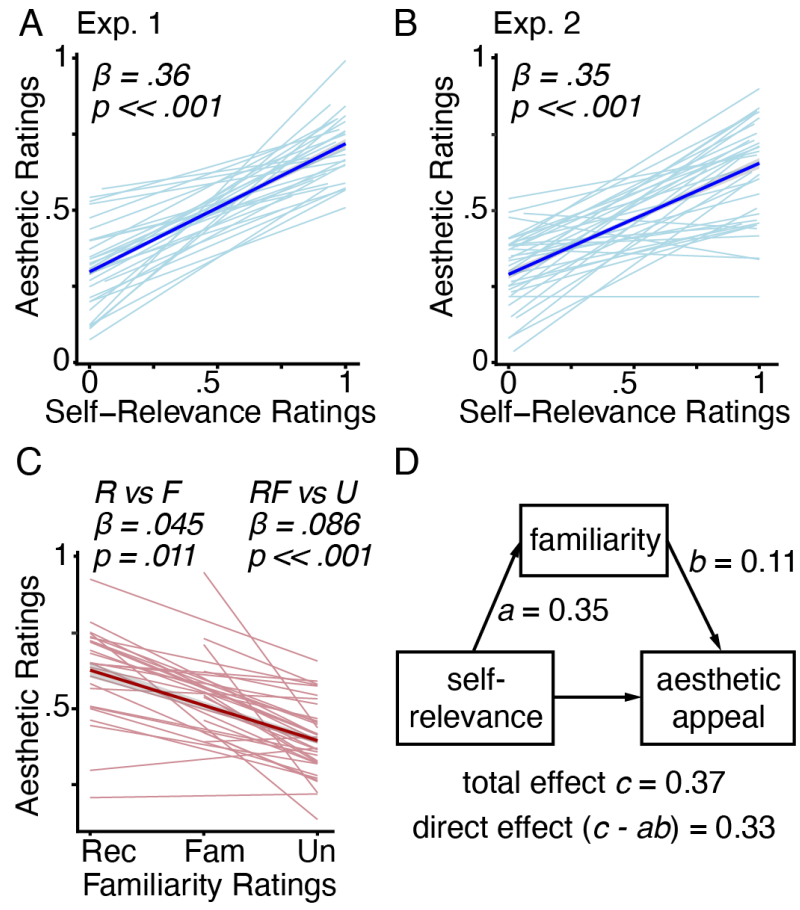

D

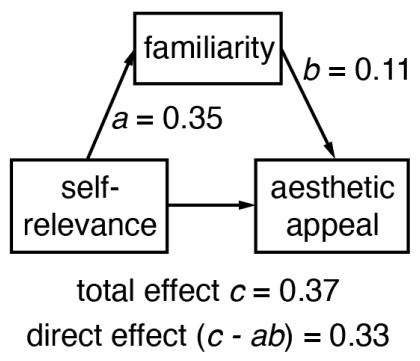

Figure 3: Predicting aesthetic ratings from ratings of self-relevance and familiarity. a) For Exp. 1, self-relevance ratings predicted aesthetic appeal with an average slope of 0.36 . b) For Exp. 2, self-relevance ratings predicted aesthetic appeal with an average slope of 0.35 . For both plots, the thick dark blue line indicates the average slope (with the standard error of the estimated slope in light gray) and the thin light blue lines show the fits for individual participants. c) In Exp. 2, familiarity also predicted ratings of aesthetic appeal, with the larger effect for ratings of Recognized or Familiar artworks versus Unfamiliar artworks (RF vs U). There was a smaller but significant effect for Recognized versus Familiar artworks (R vs F). Rec, definitely recognized; Fam, familiar; Un, unknown. d) A mediation model shows that the total effect of self-relevance on aesthetic appeal $c$ was only partially mediated by familiarity (paths $a$ and $b$ ), leaving a strong direct effect $(c-a b)$.

the mixed class (slope $=0.024,95 \% \mathrm{CI}[-0.005,0.05], \mathrm{t}[1555]=1.6, p=0.11$ ). Thus, artworks that related to core concepts of an individual's self-construct were rated as most appealing.

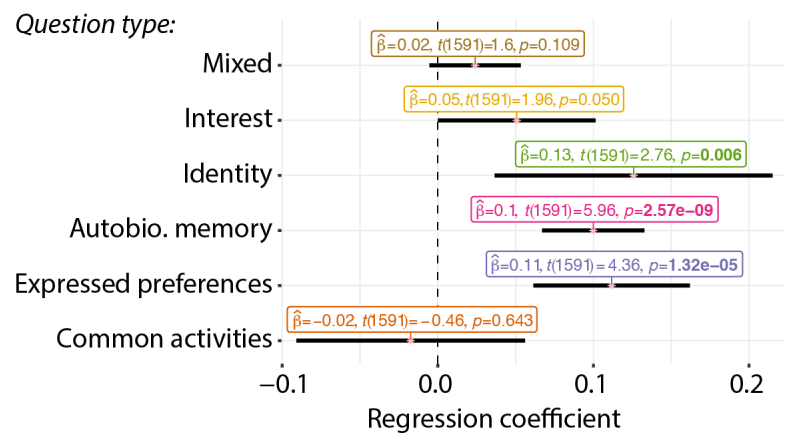

Figure 4: Artworks in the self-relevant (SR) condition that reflected specific autobiographical memories (238 items), identity (26 items), expressed preferences ( 87 items) and interests (86 items) were rated as significantly more aesthetically appealing than generated control (GC) artworks (baseline, 800 items), whereas artworks reflecting common activities (39 items), or those derived from questions reflecting "mixed" (324 items) aspects of self-relevance, were not. $\mathrm{N}=40$ participants.

From the above analyses, we conclude that the primary effect on aesthetic appeal is driven by subjective aspects of observers' responses to the paintings. We wished to determine the degree to which the perceptual effects of the style 
transfer procedure may have influenced aesthetic ratings. We therefore computed a measure of perceptual similarity between each generated artwork and its source image, based on network activation in a VGG neural network for object recognition (Learned Perceptual Image Patch Similarity LPIPS; [see 25]). This allowed for an assessment of whether the "content loss" of our artworks was related to their aesthetic appeal. On average, there was no difference in average content loss between SR and OR conditions (SR $\mu=0.564, \sigma=0.033$; OR $\mu=0.564, \sigma=0.040 ; 2$-tailed paired $\mathrm{t}(39)=0.033, p=0.97$ ). In additon, LPIPS scores were not correlated with trial-wise aesthetic ratings (for SR trials, $\mathrm{r}$ $=-0.046, p=0.19$; for OR trials, $\mathrm{r}=0.00023, p=0.99$ ). Hence, the degree to which the style transfer process rendered the novel artworks as perceptually dissimilar from the original photographs was not predictive of aesthetic appeal.

\section{Discussion}

Experiences with artwork are paradigmatic examples of how interactions with the external world can reach inside and deeply affect a person. When directly measured, aesthetic tastes in visual artwork tend to be highly individual [3, 4, 20]. Here, we show that judgments of aesthetic appeal of visual art are strongly correlated with judgments of self-relevance (Exp. 1). Furthermore, we show that novel artworks generated to contain individually-tailored selfrelevant content are rated as more aesthetically appealing than matched artworks generated for a different person (Exp. 2). Real artworks were rated as highly appealing but less self-relevant (on average), suggesting that the addition of self-relevant content can increase aesthetic ratings in a manner that is independent of the artistic skill that characterizes real artworks. Although rated familiarity did positively correlate with aesthetic appeal, the effect of self-relevance on aesthetic appeal was not mediated by familiarity. Rather, those artworks that related to core concepts of an individual's self-construct were most associated with higher aesthetic ratings. Together, these findings identify self-relevance as a key determinant of aesthetic appeal, independent of artistic skill and image features.

\section{Concerns and limitations}

Individually customized artworks generated in response to participants' responses on the 'Cultural Background and Lifestyle Questionnaire' were rated as more aesthetically appealing than either artworks generated for a different participant or a control set of artworks shown to all participants. Explicit ratings of self-relevance, collected after the ratings of aesthetic appeal, confirmed that participants found the customized artworks to be highly self relevant. In particular, artworks that related to autobiographical memories, identity and interests (to a lesser extent) were rated as more aesthetically appealing. These findings indicate that we successfully managed to manipulate the self-relevance of artworks.

We also observed higher aesthetic ratings for generated artworks whose content reflected explicitly expressed preferences (e.g. "I like Vietnamese pho soup"). While such preferences can indeed be considered a part of one's selfconstruct, in the context of the current study it was important to separate out the contribution of such preferences to avoid circularity. By doing so, it became clear that reference to aspects of the self-construct that were not simply expressed preferences also led to robust increases in appeal. On the other hand, generated artworks reflecting common activities engaged in by the participants ("What activities come to mind when you think about a rainy day?") were not rated as more appealing. However, we note that only very few items (39) contributed to this category, making it difficult to assess its reliability. Further work is needed to assess whether reference to such common activities, which do not necessarily reflect core aspects of the self construct but may instead reflect momentary goal relevance, affect aesthetic appeal.

The finding that real artworks were rated as more aesthetically appealing than the generated control artworks, even though they were rated low on self-relevance, points to the presence of something in real artworks that was not captured by the generated artworks. We suggest that the higher aesthetic appeal for real artworks may be attributable to the artistic skill reflected in these works. Nevertheless we cannot currently rule out a potential role for the larger range of styles, content and abstraction found in the set of real artworks. In comparison, the fact that the generated artworks with self-relevant content resulted in similarly high ratings of aesthetic appeal suggests that the effect of selfrelevant content is largely independent of factors related to artistic skill. Note that input styles were matched across the self-relevant and other-relevant conditions, which rules out any contribution of residual artistic differences in input styles to the observed difference in rated appeal for generated self-relevant artworks.

We found that familiarity did indeed positively predict aesthetic ratings, with the biggest effect coming from the contrast of familiar vs. unfamiliar artworks. Explicit recognition of content (versus being merely familiar) also predicted higher aesthetic appeal, but with a smaller effect. This finding is consistent with previous work showing that previous exposure can increase liking [the "mere exposure" effect [26-28] and the claim that perceptual and conceptual fluency is generally associated with greater liking [29, 30]. On the other hand, we note that there are also cases where observers show preferences for novel stimuli [28, 31]; in this data set, for example, we found that real 
artworks were rated as less familiar than self-relevant generated artworks, but also received high ratings of aesthetic appeal. Importantly, however, the overall effect of self-relevance on appeal was largely independent of familiarity. The mediation analysis (Fig. 3d) makes it clear that even when the content of an artwork was not specifically rated as familiar, the presence of content that related to core constructs of the self led to higher aesthetic appeal.

Importantly, participants were not told beforehand that the artworks they were viewing were artificially generated. For these paintings, it was not actually possible for the image to have been seen previously, and hence they were all "novel" in a strict sense (unlike for the real paintings, where it was possible, though unlikely, that they had been previously seen). Thus the wording of our instructions were constructed in a manner that allowed observers to express a feeling of familiarity with the content. Instances in which the observer found the content familiar or even explicitly recognizable (e.g. a place they had been before) could thus be reasonably marked "familiar" without the observer having to reconstruct the source of that familiarity.

\section{Functional interpretations}

Why is it that content related to one's self-construct is more likely to be experienced as aesthetically appealing? We suggest that the answer lies in the link between aesthetic appeal and information gain. Our knowledge about the world is structured hierarchically, with information about the self being at the top of that structure. Information about oneself matters more. In the social psychology literature on the self-reference effect [11], much has been made of whether the self-construct is "special" (e.g. is organized differently, relies on special mental and neural processes) in a way that reference to the self would confer an encoding advantage, or whether self-reference merely promotes deeper processing more generally [13] The generally accepted answer is that both are true [6, 12, 32], and that these two explanations are intertwined. The self-construct is special, and does seem to have some unique correlates [17, 33-36], but part of this specialness may be due to its unique position at the center of our structured knowledge about the world.

Within the broader literature on aesthetic judgments, it has been shown that higher-order semantic and associative information generally matters more than low-level perceptual features [37-41]. Particularly in the context of a predictive processing account of the mind/brain [42-44], this is consistent with theories that posit that sense-making and uncertainty reduction are experienced as pleasurable [31, 45, 46]: information gains that relate to higher nodes of a knowledge hierarchy (such as a resolution of conceptual ambiguity or a change in beliefs) allow for a greater reduction in overall uncertainty about our model of the world. Supporting evidence for this account has been reported in both the auditory [47] and visual domains [48]. Given the centrality of the self-construct, it follows that acquiring information that relates to the self, and hence has the capacity to reduce uncertainty about central aspects of our world model, leads to greater "aha" and greater pleasure than a change in beliefs or resolution of ambiguity about a non-personal object, or about a resolution of a perceptual ambiguity.

Given the importance of self-relevance for aesthetic appeal, it is relevant to ask the reverse question: are aesthetics important for one's sense of self? Recent work showing that a change in a person's aesthetic tastes are judged to alter a person's identity [49] suggest that the answer is yes.

Experiences with art can be transformative. Yet even for mundane circumstances, aesthetic factors can strongly influence behavior, mood and productivity. Our results show that there is a tight connection between aesthetic appeal and one's sense of self, and we predict that a similarly tight relationship would be observed for other forms of pleasure from comprehension, such as insight problem solving [e.g. 50] and creative inspiration [1, 51]. Beyond what these results can tell us about the psychology of aesthetic experiences, they also bear on an increasingly urgent problem in our digitally immersive world. Recommender systems, which are designed to create and deliver personalized content (e.g. TikTok, Instagram, YouTube) have become ubiquitous in our digital lives, and psychologists have flagged their great potential for problematic use [e.g. [52]. Understanding the link between self-relevance and aesthetic engagement is critical for the development of theory and policy regarding how such systems can be used safely.

\section{Methods}

\section{Experiment 1}

\section{Participants}

Thirty-three German speaking participants ( 29 female, 4 male; 30 right handed, 3 left handed) between the ages of 18 and 55 years (mean age $=28.9$ years, $\mathrm{SD}=7.3$ ) were recruited through a research participant database maintained by MPIEA and by advertisements on the institute website. Participants had normal or corrected-to-normal vision and no known neurological disorders. They were informed that the study would be about rating artworks according to how pleasing those pieces were for them; they were not aware of the study's interest in self-relevance beforehand. 
Participants signed a consent form and received monetary compensation. All recruitment and study procedures were approved by the Ethics Council of the Max Planck Society.

\section{Stimuli}

Images and instructions were presented on an ASUS VG248QE Full-HD Gaming Monitor $(60.96 \mathrm{~cm})$ computer running Windows 10 Pro (Microsoft Corporation) and MATLAB R2017a (The MathWorks) with Psychophysics Toolbox3 extensions [53](http://psychtoolbox.org). Subjects were positioned approximately $57 \mathrm{~cm}$ from the monitor.

The stimulus set consisted of 148 photos of visual artworks used in a previous study [3]. The images were originally selected from the Catalog of Art Images Online (CAMIO; note that this database was closed Dec. 2018 and is no longer accessible). The images were taken from museum collections, though commonly reproduced artworks were not included to minimize the possibility of recognition. Moreover, the set covered a variety of time periods, styles, and genres from cultures across the Americas, Europe and Asia [see 3, 22]. Images were scaled such that the largest dimension did not exceed $20^{\circ}$ of visual angle, and the area did not exceed $75 \%$ of a $20^{\circ}$ box.

For the retest sessions, 20 representative images were selected from the larger set of 148 . The practice test used 10 additional images not contained in the primary stimulus set.

\section{Procedure}

Following the informed consent procedure, participants were brought into a computer booth and instructed to sit comfortably in front of the screen and mouse. The whole experiment consisted of four blocks. Before each block, observers were given written and verbal instructions for that block. Block 1 consisted of aesthetic ratings of all 148 artworks (in random order, but with even-numbered observers getting the reverse order of odd-numbered participants). Block 2 consisted of self-relevance ratings of all 148 artworks (same order as for Block one). Observers were given 10 practice trials before Blocks 1 and 2 to familiarize themselves with the task and general nature of the artworks. Blocks 3 and 4 were 'retest' blocks for aesthetic ratings and self-relevance ratings, for a subset of 20 artworks. Within as well as between blocks, subjects were allowed breaks.

Each trial began with a fixation cross for $0.5 \mathrm{~s}$, followed by the artwork for $5 \mathrm{~s}$, then the response screen. A mid-gray background was used for all phases of the experiment.

\section{Ratings for Aesthetic Appeal}

Participants were asked to rate how aesthetically pleasing an image was for them using a continuous response scale. Participants were encouraged to answer according to their own aesthetic evaluation based on how "moved" they were by the paintings. Observers used the mouse to move a slider that appeared on the screen, marked at the ends with "N" (for "Niedrig," low) and "H" (for "Hoch," high) and were encouraged to use the full range of the scale.

\section{Ratings for Self-Relevance}

Participants were asked to rate how self-relevant an artwork was for them using a continuous response scale. Selfrelevance was defined as "the extent to which something relates to you, your experiences or your identity. These are the things and events that define you as a person." Observers used the mouse to move a slider that appeared on the screen, marked at the ends with "N" (for "Niedrig," low) and "H" (for "Hoch," high) and were encouraged to use the full range of the scale.

\section{Questionnaires}

Before the experiment, participants filled a questionnaire assessing basic demographic information (education background, age, gender, handedness, sexual orientation, diagnosed mood/psychological/neurological disorders, medication) and further education in the field of arts and aesthetics. In addition, participants completed the Aesthetic Responsiveness and Engagement Assessment [AReA; 54], a short questionnaire that identifies three major aspects of an individual's responsiveness to aesthetic experiences; the Big Five Inventory - Extra Short form [BFI-XS; [55, 56], which measures the big five personality traits; and the abbreviated Positive and Negative Affect Schedule [PANAS-X; [57, which was used to measure state affect. PANAS was applied also after experiment conduction from participant 5 onwards.

\section{Data Analysis}

Data were analyzed and visualized using using R (R Core Team, 2018). Slider responses for both aesthetic appeal and self-relevance were coded on a scale of 0 to 1 .

A reliability score was computed for both aesthetic and self-relevance ratings as the correlation between ratings of the 20 artworks shown in both test and retest blocks, separately for each participant. These two scores were then 
transformed to $z$ scores (Fisher $r$ to $z$ ), averaged, and transformed back to $r$ values to produce an average reliability score for each participant. One observer's average reliability score was below the 0.5 cutoff value and was thus removed from further analysis (final $N=32$ ).

A linear mixed model (LMM) using lmer from the lme4 package in R [58] was computed to predict ratings of aesthetic appeal (Block 1) from ratings of self-relevance (Block 2). Models were computed that included only participantspecific intercepts (Model 1), participant-specific intercepts plus slopes (Model 2), participant-specific intercepts and slopes plus image-specific intercepts (Model 3), and participant-specific intercepts plus image-specific intercepts and slopes (Model 4). Model comparison using anova revealed that while Model 3 clearly outperformed the others (log Likelihood M1 = 506, M2 = 609, M3 = 787, M3 vs. M2 $\chi^{2}(1)=358, p<2 \times 10^{-16}$ ), the addition of image-specific slopes did not lead to a significant improvement in fit $\left(\log\right.$ Likelihood M4 $=791$, M4 vs. M3 $\chi^{2}(2)=5.1, p=$ 0.08). Degrees of freedom for t-tests were computed using Satterthwaite's method, and $95 \%$ confidence intervals were computed by bootstrapping with 500 simulations using the confint.merMod function from lme 4.

To quantify the degree of shared taste and shared self-relevance across observers, leave-one-out (or "mean-minusone", MM1) agreement scores were computed after [3]. For each participant, a Pearson correlation was computed between their set of ratings for the image set and the average of all other observers. These individual correlations were then converted to z-scores (r-to-z transform), averaged, and converted back into $\mathrm{r}$ scores).

Variance partitioning was performed by first computing a multilevel model (using lmer) with random intercepts for stimuli, participants, block (test vs. retest), and the two-way interactions between these terms [59, 60]. The resulting variance partition components (VPC) were then combined to compute the proportion of repeatable variance that is "individual" versus "shared,"[59] with

$$
\text { Variance }_{\text {individual }}=\frac{V P C_{\text {participant }}+V P C_{\text {stimulusX participant }}+V P C_{\text {stimulusXblock }}}{1-V P C_{\text {error }}}
$$

and

$$
\text { Variance }_{\text {shared }}=1-\text { Variance }_{\text {individual }}
$$

\section{Experiment 2}

\section{Participants}

Based on a power analysis conducted using G*Power Software version 3.1 (Faul et al., 2007) for a two-tailed paired t-test (power $=.8$ and alpha $=.05$ ), we aimed for a sample of 45 participants, allowing for detection of an effect size of 0.45. 59 participants were recruited online via Prolific (Prolific, United Kingdom) for the initial session (see below), of which 52 were invited to take part in the main session, and 45 completed. Participants not selected for the second session were excluded because they did not provide enough answers to the Cultural Background and Lifestyle Questionnaire (see below) to allow for self-relevant stimulus creation. The participants were between 18 and 55 years old (28 male, 15 female, 2 non-identified; 41 right handed, 3 left handed, 1 ambidextrous; mean age = 29.8, SD = 8.0), fluent in German, had normal or corrected-to-normal vision and no previously known neurological disorders. They were informed that the study would be about rating artworks according to how pleasing those pieces were for them; they were not aware of the study's interest in self-relevance beforehand. Participants digitally signed a consent form and received monetary compensation. All recruitment and study procedures were approved by the Ethics Council of the Max Planck Society.

\section{Stimuli}

Each participant viewed 80 artworks, 20 in each of four conditions. The real artwork (RA) condition consisted of 20 paintings selected from the set used in Exp. 1 to cover a variety of time periods, styles, genres and cultural origin. The remaining three conditions consisted of novel artworks generated using a style transfer algorithm (see below).

The generated control (GC) artworks were the same for all participants. GC content source photographs contained a mixture of natural and manmade content, outdoor and indoor scenes, built structures and objects.

The self-relevant (SR) artworks were generated uniquely for each participant. Content source images were collected from online sources (Google Images, open source websites; minimum size of 800x600 pixels) that depicted places, objects, cuisine, animals and cultural artifacts referenced by the participant in the 'Cultural Background and Lifestyle Questionnaire.'

The other-relevant (OR) artworks consisted of the set of self-relevant artworks generated for a paired participant. The applied artistic styles (but not source content) were matched across the two paired participants to control for the effect of style. 
An initial set of 96 artworks were identified as potential style source images. In addition to the set of artworks used in Exp. 1, additional artworks were collected from online collections of the Metropolitan Museum of Art (New York), the National Gallery of Art (Washington DC) and the Rijksmuseum (Amsterdam). Of these, 56 were used as style sources for the GC, SR and OR conditions.

The images used for practice trials were a mix of real artworks and style-transferred artworks, different from those used in the main experiment.

\section{Style Transfer}

Novel generated artworks were created using the 'adaptive instance normalization' (AdaIN) method developed by Huang \& Belongie [24]. Like the original style transfer method [23], it uses a deep convolutional neural network (DCNN) to apply the 'style' of an artwork to a photograph. The general approach is to match the low- and midlevel feature statistics of the input style across the hierarchical feature representations (convolutional layers) of the DCNN-encoded content image. The network accomplishes this by first encoding both the content and style images in the feature space of an encoder network composed of the first few layers of a pre-trained VGG-19 DCNN [61]. The feature maps are then fed to the AdaIN layer, which aligns the mean and variance of the content feature maps to those of the style feature maps to produce a set of target feature maps. Finally, a decoder (randomly initialized) is trained to map from the target feature map back into image space, which generates a stylized image.

For each pair of observers, the 2 x 20 'self-relevant' content images were combined with several input styles to identify viable combinations that could be applied to an image from each of the two sets without introducing excessive blur or artifacts. Our goal was to generate images that looked, as much as possible, like actual artworks. Some styles were found to work better for certain types of content (landscape, object closeup, building, etc.), and were used more frequently. However, styles were always matched across the pair of observers, with any one style being used for only one pair of images in each pair of observers. The size of the output images were matched to the input size of the photograph, and all images were saved in jpeg format.

\section{Procedure}

On account of COVID-19 related restrictions, data was collected using the web-based Unipark Survey Platform (QuestBack GmbH, Germany). The experiment consisted of two sessions and was conducted in German. In Session 1, participants completed a set of questionnaires that included the 'Cultural Background and Lifestyle Questionnaire' used to identify self-relevant content (see below). Participants were then grouped into pairs for the generation of self-relevant and other-relevant images, making sure that their responses on this questionnaire were sufficiently divergent.

Participants invited to Session 2 answered several questionnaires and then completed four experimental blocks. In Block 1 participants rated the full set of 80 artworks for aesthetic appeal (identical instructions as in Exp. 1). In Block 2, participants rated the full set of artworks for aesthetic appeal a second time, to assess test-retest reliability. In Block 3, participants rated the full set of artworks for self-relevance (identical instructions as in Exp. 1). In Block 4, participants rated the full set of artworks for familiarity. Stimuli were presented in pseudorandom orders that distributed the four conditions evenly across the entire block and balanced the '1-back' trial history of each condition to minimize any serial order effects. No more than two repetitions of each condition were allowed in a sequence. The same order was used for all 4 blocks. Even-numbered participants were shown condition orders that were a reversal of orders for odd-numbered participants to control for the average serial position of each condition.

Each trial began with a fixation cross for $1 \mathrm{~s}$, followed by the artwork for $4 \mathrm{~s}$, then the response screen. A mid-gray background was used for all phases of the experiment.

\section{Cultural Background and Lifestyle Questionnaire}

In order to identify candidate content for the creation of self-relevant images, we designed a 20-item "Cultural Background and Lifestyle Questionnaire" that asked participants about a variety of significant locations associated with autobiographical experiences, aspects of their personal identity and personal interests in topics such as the arts, style and cuisine .

\section{Additional Questionnaires}

During the first session, participants filled out a demographic background questionnaire, the AReA [54] and the Big Five Inventory 2 - Short form [BFI-2-S; 55]. During the second session, participants completed the PANAS-X [57], and both the trait and state forms of the State-Trait Anxiety Inventory [STAI; 62, 63]. 


\section{Familiarity Judgment}

Participant were asked to indicate whether each artwork was unfamiliar, familiar, or "definitely recognized" by clicking a checkbox. Since the images had already been seen several times during the experiment, the instructions emphasized that they were to answer based on their knowledge prior to the experiment. Observers were to choose "definitely recognize" if they knew for certain that they had seen the artwork (or an image of it) before, with high confidence. They were to choose "familiar" if they had a sense that they had seen the artwork (or an image of it) before or that the content feels familiar. They were to choose "unfamiliar" if they had high confidence that they had not seen the artwork before.

\section{Data Analysis}

Data were analyzed and visualized using using R (R Core Team, 2018) and MATLAB (The MathWorks). Slider responses for both aesthetic appeal and self-relevance were coded on a scale of 0 to 100 and subsequently rescaled to between 0 and 1 .

As in Exp. 1, a test-retest score was computed (Pearson correlation) for each participant based on the first and second blocks of aesthetic ratings. Five participants with test-retest scores of $r<0.5$ were excluded from further analysis (final $N=40$ ).

LMM's for categorical effects were computed using lmer from the lme4 package in R [58] with 3 planned contrasts (SR vs. OR, RA vs. CG and SR vs. RA and degrees of freedom for $t$-tests computed using Satterthwaite's method. Additional post-hoc comparisons (e.g. OR vs. CG) were computed as Tukey contrasts using glht from the multcomp package, with adjusted p-values computed using the Holm method. Confidence intervals were computed by bootstrapping with 500 simulations using the confint.merMod function from lme4.

The LMM for prediction of aesthetic appeal from ratings of self-relevance and familiarity was performed using lmer on ratings rescaled to between 0 and 1 . Two participants were excluded, as they performed the familiarity task incorrectly, rating the images based on whether they had been seen during the session, rather than before the session (task instructions were subsequently modified to prevent this misunderstanding). First, a model was run predicting aesthetic appeal from self-relevance alone, with random intercepts for participants and condition (Model 1). We then computed two additional models using lmer predicting aesthetic appeal from ratings of self-relevance and familiarity, with random intercepts for participant and condition, both without (Model 2) and with random slopes (Model 3) for self-relevance by condition and participant. Familiarity was coded as two contrasts: "definitely recognize" and "familiar" versus "unfamiliar" (RF vs. U) and "definitely recognize" versus "familiar" (R vs. F). Model comparison using log Likelihood measures from anova revealed that the full model including both self-relevance and familiarity and random slopes for self-relevance by condition and participant performed best (Model 2 vs. Model 1 log Likelihood 272 vs. $243, \chi^{2}[2]=57, p=2.7 \times 10^{-13}$; Model 3 vs. Model $2 \log$ Likelihood 332 vs. 272, $\chi^{2}[4]=119$, $\left.p<2.2 \times 10^{-16}\right)$.

A formal mediation analysis was then computed using the mediation package in $\mathrm{R}$ to test whether the effect of selfrelevance on aesthetic appeal was mediated by familiarity, with confidence intervals computed using nonparametric bootstrapping with 1000 simulations.

\section{Sub-Classes of Self-Relevance}

The set of 20 questions from the Cultural Background and Lifestyle Questionnaire were assigned to one of five subgroups based on the nature of information sought by the question: specific autobiographical memories (238 items), aspects of personal identity (26 items), interests ( 86 items), common activities (39 items), explicitly expressed preferences (e.g. "my favorite food is Vietnamese pho soup"; 87 items). Due to their wording, some questions could not be unambiguously assigned to one of these categories, and were thus assigned to a sixth 'mixed' class (324 items). An LMM was performed using to predict aesthetic appeal for the 20 generated artworks in the self-relevant (SR) condition as a function of question class, along with participant-specific intercepts; ratings for the 20 control generated (CG) images were included as a baseline comparison, and are thus reflected in the model intercept (800 items total). 


\section{References}

[1] Dominik Welke, Isaac Purton, and Edward A Vessel. Inspired by Art: Higher Aesthetic Appeal Elicits Increased Felt Inspiration in a Creative Writing Task. Psychology of Aesthetics, Creativity, and the Arts, Advance on:1-17, 2021. doi: 10.1037/aca0000393.

[2] Kiyohito Iigaya, Sanghyun Yi, Iman A. Wahle, Koranis Tanwisuth, and John P. O'Doherty. Aesthetic preference for art can be predicted from a mixture of low- and high-level visual features. Nature Human Behaviour, 2021. doi: 10.1038/s41562-021-01124-6.

[3] Edward A. Vessel, Natalia Maurer, Alexander H. Denker, and G. Gabrielle Starr. Stronger shared taste for natural aesthetic domains than for artifacts of human culture. Cognition, 179(June):121-131, 2018. ISSN 0010-0277. doi: 10.1016/j.cognition.2018.06.009.

[4] Helmut Leder, Juergen Goller, Tanya Rigotti, and Michael Forster. Private and shared taste in art and face appreciation. Frontiers in Human Neuroscience, 10(April):1-7, 2016. ISSN 1662-5161. doi: 10.3389/fnhum. 2016.00155.

[5] Daryl J. Bem. Self-perception theory. In B. Leonard, editor, Advances in Experimental Social Psychology, pages 1-62. Academic Press, San Diego, 1972.

[6] Dylan D. Wagner, James V. Haxby, and Todd F. Heatherton. The representation of self and person knowledge in the medial prefrontal cortex. Wiley Interdisciplinary Reviews: Cognitive Science, 3(4):451-470, 2012. ISSN 19395078. doi: 10.1002/wcs.1183.

[7] Hazel Markus. Self-schemata and processing information about the self. Journal of Personality and Social Psychology, 35(2):63-78, 1977. doi: 10.1037/0022-3514.35.2.63.

[8] Geoffrey T. Fong and Hazel Markus. Self-schemas and judgments about others. Social Cognition, 1(3):191-204, 1982. doi: 10.1521/soco.1982.1.3.191.

[9] John A. Bargh and Roman D. Thein. Individual construct accessibility, person memory, and the recall-judgment link. The case of information overload. Journal of Personality and Social Psychology, 49(5):1129-1146, 1985. ISSN 00223514. doi: 10.1037/0022-3514.49.5.1129.

[10] Hazel Markus, Jeanne Smith, and Richard L. Moreland. Role of the self-concept in the perception of others. Journal of Personality and Social Psychology, 49(6):1494-1512, 1985. ISSN 00223514. doi: 10.1037/0022-3514.49.6.1494.

[11] T.B. Rogers, N.A. Kuiper, and W.S. Kirker. Self-reference and the encoding of personal information. Journal of Personality and Social Psychology, 35(9):677-688, 1977. ISSN 01475916. doi: 10.1037/0022-3514.35.9.677.

[12] Cynthia S Symons and T Blair. The self-reference effect in memory : A meta-analysis. Psychological Bulletin, 121(3):371-394, 1997. doi: 10.1037/0033-2909.121.3.371.

[13] Stanley B. Klein and Judith Loftus. "The nature of self-referent encoding: The contributions of elaborative and organizational processes": Correction to Klein and Loftus. Journal of Personality and Social Psychology, 55(6): 881-881, 1988. ISSN 0022-3514. doi: 10.1037/h0090411.

[14] Helmut Leder, Benno Belke, Andries Oeberst, and Dorothee Augustin. A model of aesthetic appreciation and aesthetic judgments. British Journal of Psychology, 95:489-508, 2004.

[15] Matthew Pelowski, Patrick S. Markey, Michael Forster, Gernot Gerger, and Helmut Leder. Move me, astonish me... delight my eyes and brain: The Vienna Integrated Model of top-down and bottom-up processes in Art Perception (VIMAP) and corresponding affective, evaluative, and neurophysiological correlates. Physics of Life Reviews, 1:1-46, 2017. ISSN 15710645. doi: 10.1016/j.plrev.2017.02.003.

[16] J R Andrews-Hanna, J S Reidler, J Sepulcre, R Poulin, and R L Buckner. Functional-Anatomic Fractionation of the Brain's Default Network. Neuron, 65(4):550-562, 2010. doi: 10.1016/j.neuron.2010.02.005.

[17] J M Moran, T F Heatherton, and W M Kelley. Modulation of cortical midline structures by implicit and explicit self-relevance evaluation. Soc. Neurosci., 4(3):197-211, 2009. doi: 10.1080/17470910802250519. URL http: //www.ncbi.nlm.nih.gov/pubmed/19424905

[18] Arnaud D’Argembeau, David Stawarczyk, Steve Majerus, Fabienne Collette, Martial Van der Linden, Dorothee Feyers, Pierre Maquet, and Eric Salmon. The Neural Basis of Personal Goal Processing When Envisioning Future Events. Journal of Cognitive Neuroscience, 22(8):1701-1713, 2010. doi: doi:10.1162/jocn.2009.21314. URL http: //www . mitpress journals .org/doi/abs/10.1162/jocn.2009.21314.

[19] Edward A Vessel, G Gabrielle Starr, and Nava Rubin. The brain on art: intense aesthetic experience activates the default mode network. Frontiers in Human Neuroscience, 6(66):1-17, 2012. doi: 10.3389/fnhum.2012.00066. 
[20] Edward A Vessel, G Gabrielle Starr, and Nava Rubin. Art reaches within: aesthetic experience, the self and the default mode network. Frontiers in Neuroscience, 7(7 DEC), 2013. doi: 10.3389/fnins.2013.00258.

[21] Amy M. Belfi, Edward A. Vessel, Aenne Brielmann, Ayse Ilkay Isik, Anjan Chatterjee, Helmut Leder, Denis G. Pelli, and G. Gabrielle Starr. Dynamics of aesthetic experience are reflected in the default-mode network. NeuroImage, 188:584-597, mar 2019. ISSN 1053-8119. doi: 10.1016/J.NEUROIMAGE.2018.12.017.

[22] Edward A Vessel, Ayse Ilkay Isik, Amy M Belfi, Jonathan L Stahl, and G Gabrielle Starr. The default-mode network represents aesthetic appeal that generalizes across visual domains. Proceedings of the National Academy of Sciences, sep 2019. doi: 10.1073/pnas.1902650116.

[23] Leon A. Gatys, Alexander S. Ecker, and Matthias Bethge. Image Style Transfer Using Convolutional Neural Networks. Proceedings of the IEEE Computer Society Conference on Computer Vision and Pattern Recognition, 2016-Decem:2414-2423, 2016. ISSN 10636919. doi: 10.1109/CVPR.2016.265.

[24] Xun Huang and Serge Belongie. Arbitrary style transfer in real-time with adaptive instance normalization. 5th International Conference on Learning Representations, ICLR 2017 - Workshop Track Proceedings, 2017.

[25] Cem Uran, Alina Peter, Andreea Lazar, William Barnes, Johanna Klon-lipok, Katharine A Shapcott, Rasmus Roese, Pascal Fries, Wolf Singer, and Martin Vink. Predictive coding of natural images by V1 activity revealed by self-supervised deep neural networks. bioRxiv, 2021.

[26] B Zajonc, Robert. Attitudinal effects of mere exposure. Journal of Experimental Social Psychology, 9(2):1-28, 1968. ISSN 10960465. doi: 10.1016/0022-1031(71)90078-3.

[27] R F Bornstein. Exposure and Affect - Overview and Meta-Analysis of Research, 1968-1987. Psychological Bulletin, 106(2):265-289, 1989.

[28] Junghyun Park, Eiko Shimojo, and Shinsuke Shimojo. Roles of familiarity and novelty in visual preference judgments are segregated across object categories. Proceedings of the National Academy of Sciences, 107 (33):14552-14555, 2010. doi: 10.1073/pnas.1004374107. URL http://www.pnas.org/content/107/33/ 14552.abstract

[29] R Reber, P Winkielman, and N Schwarz. Effects of perceptual fluency on affective judgments. Psychological Science, 9(1):45-48, 1998.

[30] Rolf Reber, Norbert Schwarz, and Piotr Winkielman. Processing fluency and aesthetic pleasure: is beauty in the perceiver's processing experience? Personality and Social Psychology Review, 8(4):364-382, 2004.

[31] Irving Biederman and Edward A Vessel. Perceptual Pleasure and the Brain. American Scientist, 94(3): 247-253, 2006. ISSN 0003-0996. doi: 10.1511/2006.3.247. URL http://www.americanscientist. org/issues $/$ issue . aspx?id=995\&y=0\&no=\&content=true\&page=4\&css=print\%5Cnpapers2 : //publication/uuid/593510F5-A7F5-47E2-837F-3B077E4EB0A9.

[32] Seth J. Gillihan and Martha J. Farah. Is self special? A critical review of evidence from experimental psychology and cognitive neuroscience. Psychological Bulletin, 131(1):76-97, 2005. ISSN 00332909. doi: 10.1037/0033-2909.131.1.76.

[33] J M Moran, C N Macrae, T F Heatherton, C L Wyland, and W M Kelley. Neuroanatomical evidence for distinct cognitive and affective components of self. Journal of Cognitive Neuroscience, 18(9):1586-1594, 2006.

[34] R. Nathan Spreng and Cheryl L. Grady. Patterns of Brain Activity Supporting Autobiographical Memory, Prospection, and Theory of Mind, and Their Relationship to the Default Mode Network. Journal of Cognitive Neuroscience, 22(6):1112-1123, 2010. ISSN 0898-929X. doi: 10.1162/jocn.2009.21282. URL http://www.mitpressjournals.org/doi/10.1162/jocn.2009.21282

[35] Jessica R. Andrews-Hanna, Jonathan Smallwood, and R. Nathan Spreng. The default network and self-generated thought: Component processes, dynamic control, and clinical relevance. Annals of the New York Academy of Sciences, 1316(1):29-52, 2014. ISSN 17496632. doi: 10.1111/nyas.12360.

[36] Vadim Axelrod, Geraint Rees, and Moshe Bar. The default network and the combination of cognitive processes that mediate self-generated thought. 1(December), 2017.

[37] Edward A Vessel and Nava Rubin. Beauty and the beholder: Highly individual taste for abstract, but not realworld images. Journal of Vision, 10(2):1-14, 2010. doi: 10.1167/10.2.18. URL http://www.ncbi.nlm.nih . gov/pubmed/20462319

[38] Astrid Schepman, Paul Rodway, and Sarah J Pullen. Greater cross-viewer similarity of semantic associations for representational than for abstract artworks. Journal of Vision, 15:1-6, 2015. doi: 10.1167/15.14.12.doi. 
[39] Astrid Schepman, Paul Rodway, Sarah J Pullen, and Julie Kirkham. Shared liking and association valence for representational art but not abstract art. Journal of Vision, 15:1-10, 2015. ISSN 1534-7362. doi: 10.1167/15.5. 11.doi.

[40] Astrid Schepman and Paul Rodway. Shared Meaning in Representational and Abstract Visual Art: An Empirical Study. Psychology of Aesthetics, Creativity, and the Arts, 2019. ISSN 1931390X. doi: 10.1037/aca0000279.

[41] S E Palmer and K B Schloss. An ecological valence theory of human color preference. Proc Natl Acad Sci U S A, 107(19):8877-8882, 2010.

[42] Andy Clark. Whatever next? Predictive brains, situated agents, and the future of cognitive science. Behavioral and Brain Sciences, 36(3):181-204, 2013. ISSN 14691825. doi: 10.1017/S0140525X12000477.

[43] Karl Friston. Hierarchical models in the brain. PLoS Computational Biology, 4(11), 2008. ISSN 1553734X. doi: 10.1371/journal.pcbi.1000211.

[44] Rajesh P.N. Rao and Dana H. Ballard. Predictive coding in the visual cortex: A functional interpretation of some extra-classical receptive-field effects. Nature Neuroscience, 2(1):79-87, 1999. ISSN 10976256. doi: $10.1038 / 4580$.

[45] Jürgen Schmidhuber. Formal Theory of Creativity Intrinsic Motivation ( 1990-2010 ). IEEE Transactions on autonomous mental development, 2(3):1-18, 2010.

[46] Felix Schoeller and Leonid Perlovsky. Aesthetic chills: Knowledge-acquisition, meaning-making, and aesthetic emotions. Frontiers in Psychology, 7(AUG):1-16, 2016. ISSN 16641078. doi: 10.3389/fpsyg.2016.01093.

[47] Vincent K M Cheung, Peter M C Harrison, Lars Meyer, Marcus T Pearce, John-Dylan Haynes, and Stefan Koelsch. Uncertainty and Surprise Jointly Predict Musical Pleasure and Amygdala, Hippocampus, and Auditory Cortex Activity. Current Biology, 29(23):4084-2092.e4, 2019. doi: 10.1016/j.cub.2019.09.067. URL http: //www.ncbi.nlm.nih.gov/pubmed/31708393.

[48] Sander Van de Cruys, Claudia Damiano, Yannick Boddez, Magdalena Król, Lore Goetschalckx, and Johan Wagemans. Visual affects: Linking curiosity, Aha-Erlebnis, and memory through information gain. Cognition, 212(September 2020):104698, 2021. ISSN 00100277. doi: 10.1016/j.cognition.2021.104698.

[49] Joerg Fingerhut, Javier Gomez-Lavin, Claudia Winklmayr, and Jesse J. Prinz. The Aesthetic Self. The Importance of Aesthetic Taste in Music and Art for Our Perceived Identity. Frontiers in Psychology, 11(March):1-18, 2021. ISSN 16641078. doi: 10.3389/fpsyg.2020.577703.

[50] Robert J Sternberg and Janet E Davidson, editors. The nature of insight. The MIT Press, Cambridge, MA, US, 1995. ISBN 0-262-19345-0 (Hardcover).

[51] Todd M. Thrash and Andrew J. Elliot. Inspiration as a Psychological Construct. Journal of Personality and Social Psychology, 84(4):871-889, 2003. ISSN 00223514. doi: 10.1037/0022-3514.84.4.871.

[52] Conghui Su, Hui Zhou, Liangyu Gong, Binyu Teng, Fengji Geng, and Yuzheng Hu. Viewing personalized video clips recommended by TikTok activates default mode network and ventral tegmental area. NeuroImage, 237 (April):118136, 2021. ISSN 10538119. doi: 10.1016/j.neuroimage.2021.118136.

[53] David H. Brainard. The Psychophysics Toolbox. Spatial Vision, 1997. ISSN 01691015. doi: 10.1163/ $156856897 X 00357$.

[54] Wolff Schlotz, Sebastian Wallot, Diana Omigie, Michael D Masucci, Sonja C Hoelzmann, and Edward A Vessel. The Aesthetic Responsiveness Assessment (AReA): A Screening Tool to Assess Individual Differences in Responsiveness to Art in English and German. Psychology of Aesthetics, Creativity, and the Arts, Advance on (August 20):1-15, 2020. doi: 10.1037/aca0000348.

[55] Christopher J. Soto and Oliver P. John. Short and extra-short forms of the Big Five Inventory-2: The BFI-2-S and BFI-2-XS. Journal of Research in Personality, 68:69-81, 2017. ISSN 10957251. doi: 10.1016/j.jrp.2017.02.004. URL http://dx.doi.org/10.1016/j.jrp.2017.02.004

[56] Christopher J. Soto and Oliver P. John. The next Big Five inventory. Journal of Personality and Social Psychology, 113(1):117-143, 2017. doi: 10.2214/ajr.170.6.9609152.

[57] David Watson and L. A. Clark. The PANAS-X: Manual for the Positive and Negative Affect Schedule -Expanded Form THE PANAS-X Manual for the Positive and Negative Affect Schedule -Expanded Form. Department of Psychological and Brain Sciences Publications, page 28, 1999. doi: 10.17077/48vt-m4t2.

[58] Douglas Bates, Martin Mächler, Ben Bolker, and Steve Walker. Fitting Linear Mixed-Effects Models Using lme4. Journal of Statistical Software, 67(1), 2015. ISSN 1548-7660. doi: 10.18637/jss.v067.i01. 
[59] Joel E. Martinez, Friederike Funk, and Alexander Todorov. Quantifying idiosyncratic and shared contributions to judgment. Behavior Research Methods, 52(4):1428-1444, 2020. ISSN 15543528. doi: 10.3758/ s13428-019-01323-0. URL https://doi.org/10.31234/osf.io/6vr8z.

[60] Clare A.M. Sutherland, Nichola S. Burton, Jeremy B. Wilmer, Gabriëlla A.M. Blokland, Laura Germine, Romina Palermo, Jemma R. Collova, and Gillian Rhodes. Individual differences in trust evaluations are shaped mostly by environments, not genes. Proceedings of the National Academy of Sciences of the United States of America, 117(19):10218-10224, 2020. ISSN 10916490. doi: 10.1073/pnas.1920131117.

[61] Karen Simonyan and Andrew Zisserman. Very deep convolutional networks for large-scale image recognition. 3rd International Conference on Learning Representations, ICLR 2015 - Conference Track Proceedings, pages $1-14,2015$.

[62] C. D. Spielberger, R. L. Gorsuch, and R. E. Lushene. The State-Trait Anxiety Inventory. MANUAL, pages 1-23, 1970.

[63] C D Spielberger, R L Gorsuch, R Lushene, P R Vagg, and G A Jacobs. Manual for the State-Trait Anxiety Inventory. Consulting Psychologists Press, Palo Alto, CA, 1983. 